H I G H L I G H T S

HUMAN GENETICS

\section{Rare insights into common disease}

Although Bardet-Biedl syndrome (BBS) is a rare, recessive disease, it is of interest to human geneticists because its diverse clinical characteristics include diabetes, obesity, hypertension and congenital heart disease. But BBS is also genetically complex, involving six distinct loci (BBS1-BBS6). The characterization of $B B S 6$ had raised expectations that the BBS proteins might act in a chaperone complex, although the recently discovered $B B S 2$ encodes a protein of unknown function. Now, Val Sheffield and colleagues have discovered $B B S 4$, which encodes a protein with homology to $O$-linked $N$-acetylglucosamine transferase (OGT), adding to the questions that surround the functions of BBS proteins.

Myktyn et al. located BBS4, which had been previously mapped to chromosome 15, by haplotype analysis in three consanguineous BBS4 (chromosome 15) pedigrees. Sequence from the candidate interval revealed several candidate genes, one of which was mutated in five of six BBS4 families. The mutation spectrum included a homozygous $\mathrm{G} \rightarrow \mathrm{C}$ transversion in exon 12, predicted to cause an Arg to Pro substitution, which segregated completely with BBS in a large Bedouin kindred. Other mutations were predicted to disrupt splicing. Interestingly, a 6-kb deletion that encompassed two exons of BBS4 was found in two unrelated BBS families - affected individuals had identical breakpoints in Alu elements flanking the deletion. The authors speculate that similar $A l u$-associated BBS4 mutations might be found in other BBS families because the high frequency of $A l u$ repeats in $B B S 4$ could predispose it to mutation by unequal homologous recombination.

It still remains to be shown how BBS4 contributes to the BBS phenotype, but there are some clues as to its function. In humans, OGT has been implicated in insulin resistance and diabetes, and the BBS4 protein has a motif that facilitates protein interactions. There are still more pieces of the BBS puzzle to come, and fitting together the BBS proteins into functional pathways is clearly an important goal for this field. In doing so, it will hopefully reveal rare insights into the molecular mechanisms that contribute to the common diseases of diabetes, obesity and hypertension, and to congenital heart disease.

Jane Alfred

\section{(2) References and links}

ORIGINAL RESEARCH PAPER Myktyn, K. et al. Identification of the gene that, when mutated, causes the human obesity syndrome BBS4. Nature Genet. 28, 188-191 (2001)

FURTHER READING Slavotinek, A. M. et al. Mutations in MKKS cause Bardet-Biedl syndrome. Nature Genet. 26, 15-16 (2000) | Katsanis, M. et al. Mutations in MKKS cause obesity, retinal dystrophy and renal malformations associated with Bardet-Biedl syndrome. Nature Genet. 26, 67-70 (2000) | Nishimura, D. Y. et al. Positional cloning of a novel gene on chromosome 16q causing Bardet-Biedl syndrome (BBS2). Hum. Mol. Genet. 10, 865-874 (2001)

WEB SITE Bardet-Biedl web links

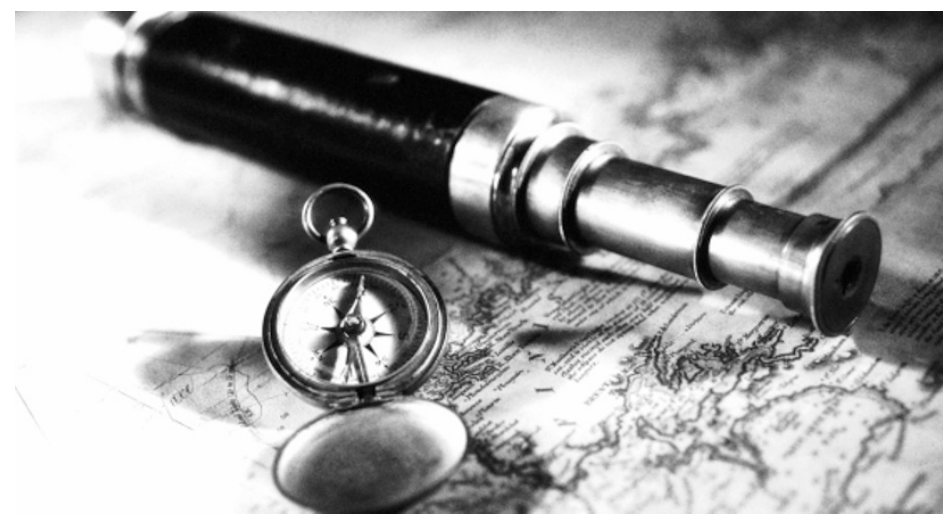

\section{IN BRIEF}

\author{
TECHNOLOGY
}

RNomics: an experimental approach that identifies 201 candidates for novel, small, non-messenger RNAs in mouse.

Huttenhofer, A. et al. EMBO J. 20, 2943-2953 (2001)

Small non-messenger (snm) RNAs have a role in cellular physiology, translation and splicing. Because they are difficult to find by the computational analysis of genomic sequences, Huttenhoffer et al. took an EST-like approach to isolate 50-500nucleotide-long expressed RNA sequences from mouse cells. Among the 201 novel candidates found were small nucleolar RNAs that guide RNA ribose methylation and pseudouridylation and those that modify spliceosomal small nuclear RNA. Some snmRNAs were tissue specific, indicating a new role for snmRNAs in gene expression.

\section{DEVELOPMENTAL BIOLOGY}

A crucial component of the endoderm formation pathway, CASANOVA is encoded by a sox-related gene. Dickmeis, T. et al. Genes Dev. 15, 1487-1492 (2001)

casanova encodes a novel Sox-related protein necessary and sufficient for early endoderm formation in zebrafish.

Kikuchi, Y. et al. Genes Dev. 15, 1493-1505 (2001)

Several zebrafish mutations disrupt endoderm formation, many of which are in the Nodal signalling pathway. Of these endoderm mutants, casanova (cas) has the most severe endoderm phenotype - it fails to express markers of endoderm differentiation or formation and does not form gut tissue. These studies identify cas as a novel sox-related gene - Kikuchi et al. found it by a positional-cloning and candidate-gene approach, and Dickmeis et al. in a subtractive screen for Nodalresponsive genes - and show that it is the principal effector of Nodal signalling in zebrafish endoderm formation. For example, Kikuchi et al. report that cas can induce sox 17 expression, an early endoderm marker, in mutants that cannot respond to Nodal signalling, and that its ectopic expression causes mesodermal cells to switch fate to endoderm.

\section{TECHNOLOGY}

\section{Microarrays of cells expressing defined cDNAs.}

Ziauddin, J. \& Sabatini, D. M. Nature 411, 107-110 (2001)

Rapid analysis of gene function is a priority in the postgenomic era. This paper reports the development of a new microarray technique that involves culturing mammalian cells on glass slides that have been printed with arrays of cDNAs in expression vectors. These 'living arrays' can be screened for cellular phenotypes because the cells become transfected with the expression constructs. In a test experiment, the authors identified proteins involved in tyrosine kinase signalling, apoptosis and cell signalling. This approach could be used to identify drug targets and new proteins that alter cell physiology. 